\title{
Pharmacogenetics in the treatment of pre- eclampsia: current findings, challenges and perspectives
}

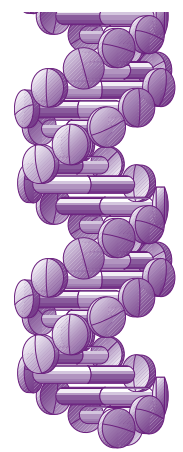

Pre-eclampsia (PE) is defined as pregnancy-induced hypertension and proteinuria, and is a major cause of maternal and perinatal morbidity and mortality. A large subgroup of pregnant women with PE is nonresponsive to antihypertensive drugs, including methyldopa, nifedipine and hydralazine. Pharmacogenomics may help to guide the individualized therapy for this nonresponsive subgroup. However, just a few pharmacogenetic studies examined the effects of genetic polymorphisms on response to antihypertensive drugs in $\mathrm{PE}$, and the criteria of responsiveness used to define responsive or nonresponsive subgroups to antihypertensive therapy should be replicated by others. We review these gene-drugs interactions, novel approaches to pharmacogenomics research and potential novel drugs for PE therapy. Finally, we discuss the challenges and perspectives of pharmacogenetics in the treatment of PE.

First draft submitted:16 December 2016; Accepted for publication: 6 February 2017; Published online: 30 March 2017

Keywords: antihypertensive therapy $\bullet$ genotypes $\bullet$ gestational hypertension $\bullet$ haplotypes - pharmacogenetics $\bullet$ pharmacogenomics $\bullet$ polymorphisms $\bullet$ pre-eclampsia

Hypertensive disorders of pregnancy (HDP) are defined in accordance to the National High Blood Pressure Education Program Working Group on High Blood Pressure in Pregnancy [1]. Gestational hypertension $(\mathrm{GH})$ is defined as systolic blood pressure of $\geq 140 \mathrm{mmHg}$ or diastolic blood pressure of $\geq 90 \mathrm{mmHg}$ at two or more measurements after 20 weeks of gestation, and returning to normal by 12 weeks postpartum. Preeclampsia (PE), defined as GH plus significant proteinuria ( $\geq 0.3 \mathrm{~g}$ per $24 \mathrm{~h}$ ), is a multisystem disorder that can affect maternal brain, kidneys, liver and the blood clotting system [2]. PE has implications for future maternal health [3], in particular an increased risk of cardiovascular disease [4,5]. Notably, $\mathrm{PE}$ is a major cause of maternal and perinatal morbidity and mortality, with prevalence of $0.2-9.2 \%$ worldwide $[3,6]$.

Antihypertensive therapy is mandatory for high blood pressure control in PE [2], and includes methyldopa, nifedipine, hydralazine and labetalol. These antihypertensive drugs allow the prolongation of gestation, thereby decreasing fetal and maternal adverse outcomes $[7,8]$. However, a large subgroup of pregnant women with $\mathrm{PE}$ is nonresponsive to antihypertensive therapy and is associated with the worst clinical parameters, according to the criteria of responsiveness defined in our pharmacogenetic studies (Box 1) [9-14]. Importantly, this nonresponsive subgroup could benefit from a more individualized treatment and monitoring. These findings suggest that the use of pharmacogenomics may maximize the treatment outcome by reducing maternal and fetal morbidity and mortality associated with PE, as well as reducing adverse events associated with pharmacological therapy [15].

However, there are a few studies focusing on the pharmacogenetics in the treatment of PE, which examined the association of polymorphisms in candidate genes for PE
Marcelo R Luizon ${ }^{1,2}$, Ana CT Palei $^{3,4}$, Ricardo C Cavalli ${ }^{5}$ \& Valeria C Sandrim*,1

'Department of Pharmacology, Institute of Biosciences of Botucatu, Universidade Estadual Paulista (UNESP), Botucatu, Sao Paulo 18680-000, Brazil ${ }^{2}$ Department of General Biology, Institute of Biological Sciences, Federal University of Minas Gerais, Belo Horizonte, Minas Gerais 31270-901, Brazil ${ }^{3}$ Department of Surgery, Division of Pediatric \& Congenital Heart Surgery, University of Mississippi Medical Center, Jackson, MS 39216, USA

${ }^{4}$ Cardiovascular-Renal Research Center, University of Mississippi Medical Center, Jackson, MS 39216, USA

${ }^{5}$ Department of Gynecology \& Obstetrics, Ribeirao Preto Medical School, University of Sao Paulo, Ribeirao Preto, Sao Paulo 14049-900, Brazil *Author for correspondence: Tel.: +551438800228 Fax: +55 1438153744 valeria.sandrim@ibb.unesp.br 
Box 1. The criteria of responsiveness to antihypertensive therapy in gestational hypertension and pre-eclampsia used by our group.

- Responsiveness to antihypertensive therapy was based on the evaluation of clinical and laboratory parameters (see below) in response to the administration of antihypertensive drugs. The initial antihypertensive drug of choice was methyldopa (1000-1500 mg per day), followed by nifedipine (40-60 mg per day), which was added in case of lack of significant responses to methyldopa. Hydralazine (5-30 mg) was used only in cases of hypertensive crisis. One of the following clinical and laboratory outcomes were considered to classify a patient as nonresponsive to the antihypertensive therapy:

- Clinical symptoms including blurred vision, persistent headache or scotomata, persistent right upper quadrant or epigastric pain;

- Systolic blood pressure above $140 \mathrm{mmHg}$ and diastolic blood pressure above $90 \mathrm{mmHg}$, as assessed by the blood pressure curve;

- Hemolysis, elevated liver enzymes and a low platelet count syndrome; or proteinuria >2.0 g per $24 \mathrm{~h}$; creatinine $>1.2 \mathrm{mg}$ per $100 \mathrm{ml}$ or blood urea nitrogen $>30 \mathrm{mg}$ per $100 \mathrm{ml}$; aspartate aminotransferase $>70 \mathrm{Ul}^{-1}$ and alanine aminotransferase $>60 \mathrm{Ul}^{-1}$; and

- Fetal hypoactivity or nonreactive fetus, as revealed by cardiotocography; intrauterine growth restriction, oligoamnio, abnormal biophysical profile score and Doppler velocimetry abnormalities, as evaluated by ultrasound.

with subgroups of patients who are responsive or nonresponsive to antihypertensive therapy [9-14]. Here, we briefly discuss about the pathophysiology and the genetics of PE. We review the current findings from the available pharmacogenetic studies [9-14] (Table 1), novel approaches to pharmacogenomics research in $\mathrm{PE}$ and potential novel drugs for PE therapy. Finally, we also discuss the challenges and perspectives of pharmacogenetics in the treatment of PE.

\section{Pathophysiology of pre-eclampsia}

Although the exact mechanisms remain unclear, placental ischemia and hypoxia is implicated as a pivotal causative factor in the pathophysiology of PE [16]. A two-stage disorder model has been proposed for PE [17]. The first stage is marked by reduced placental perfusion, secondary to abnormal implantation and development of placental vasculature. The second stage is depicted by placental release of detrimental factors into the maternal circulation that prompts widespread endothelial dysfunction, which in turn leads to the clinical symptoms of PE. Both stages need to occur for PE to be manifested. In addition, they interact with maternal constitutional factors (genetic, behavioral or environmental) resulting in different PE subtypes [17]. Recent research has revealed glimpses into pathways playing a role in either one or both stages.

Early on pregnancy, fetal-derived cytotrophoblasts migrate toward the decidua and myometrium to invade and replace the endothelial cells of uterine spiral arteries. This process results in the conversion of highresistance, small-diameter vessels into high capacitance, large-diameter vessels to accommodate proper delivery of maternal blood to the forming fetal-placental unit. Degradation and remodeling of the uterine extracellular matrix is required for a successful trophoblastic invasion, angiogenesis and embryogenesis [18]. Indeed, reduced vascular remodeling of uterine-placental tissues and decreased invasion of cytotrophoblasts into spiral arteries were shown in placentas from pregnancies with PE [19]. Failure in any step of the process of invasion and remodeling of uterine spiral arteries may lead to inadequate delivery of oxygen and nutrients to the fetal-placental unit, triggering the secretion of signals from the placenta to overcome the restricted blood flow $[20,21]$.

Key studies into the placental factors related to the etiology of PE have focused on the interplay between pro- and antiangiogenic proteins. sFlt- 1 is a splice variant of the VEGFR-1 in which the cytoplasmic and transmembrane domains are post-transcriptionally excised. sEng is a truncated form of endoglin. While sFlt-1 antagonizes the proangiogenic actions of VEGF and PlGF, sEng inhibits the signaling of TGF- $\beta$, by sequestering free protein in the circulation and thus hindering protein interaction with their respective receptors [22]. Circulating levels of sFlt-1 and sEng were found increased in PE [23-25]. Notably, these two antiangiogenic factors seem to be altered several weeks before the onset of the clinical symptoms and are positively correlated with PE severity [26,27].

The shedding of placental factors into maternal circulation was shown to be increased in PE [28], and may activate peripheral immune cells, exacerbating the normal inflammatory response evoked during gestation. Activated T lymphocytes can contribute to the development of hypertension by secreting various cytokines, such as TNF- $\alpha$ and IL-6, and by stimulating B lymphocytes to produce autoantibodies [29]. Notably, PE patients show increased and decreased circulating levels of pro- and anti-inflammatory cytokines, respectively [30,31]. Autoantibodies against the AGTR1, which 
were consistently found to be elevated in PE, may also play a role in enhancing the maternal vascular sensitivity to the vasoconstriction actions of Ang II [32,33].

Many of the factors generated through placental ischemia and hypoxia target the maternal vascular endothelium, and activate pathways that cause increased total peripheral resistance, decreased renal excretory function, and ultimately hypertension [34]. The major mediators linked to endothelial dysfunction in PE are the vasoconstrictor peptide ET-1, the vasodilator gas nitric oxide (NO) and oxidative stress. Elevated ET-1 and reduced NO metabolites levels were shown in the circulation of PE patients [25,35-37]. Notably, these three molecular mechanisms of endothelial dysfunction, along with VEGF, were shown to be strictly connected to each other during hypertension $[38,39]$.

Aberrant placental and circulating levels of MMPs were also reported in PE [40]. Increased MMP activity upon vasoactive molecules was shown to generate vasoconstrictors and degrade vasodilators, thereby causing exaggerated vasoconstriction and hypertension [41-43]. Interestingly, activated MMPs may also contribute to endothelial dysfunction via proteolysis of cell surface receptors that mediate vasodilatation, such as VEGFR-2 [44,45].
We therefore speculate that MMPs, NO bioavailability, oxidative stress and inflammation interact to promote hypertension during pregnancy. However, this suggestion remains to be proven. Notably, these pathophysiological processes may be further explored to reveal potential therapeutic targets.

\section{Genetics of pre-eclampsia}

The identification of genetic variants that affect PE risk could substantially aid the understanding of its susceptibility, and therefore its prevention and treatment [46]. However, candidate gene studies are limited by the incomplete understanding of the pathophysiology of PE [15]. Over 70 candidate genes selected on the basis of prior knowledge from pathophysiological mechanisms in PE have been investigated, such as vasoactive proteins, thrombophilia, oxidative stress, immune and inflammatory mediators. However, no single candidate gene has been accepted as a causal gene for PE [15]. Genome-wide association studies were also performed to identify maternal SNPs and copy-number variants associated with PE. Two studies found no SNP associations that reached genome-wide significance, and copy-number variants on regions that warrant further replication $[47,48]$. Other study found

Table 1. Pharmacogenetics studies on the response to antihypertensive therapy in patients with pre-eclampsia and gestational hypertension.

\begin{tabular}{|c|c|c|c|c|}
\hline Genes & Polymorphisms & Patients: subgroups ${ }^{\dagger}(n)$ & Summary findings & Ref. $^{*}$ \\
\hline NOS3 & $\begin{array}{l}\text { T-786C (rs2070744), } \\
\text { Glu298Asp (rs1799983), } \\
\text { VNTR a/b in intron } 4\end{array}$ & $\begin{array}{l}\text { PE: R (82) and NR (70); } \\
\text { GH: R (127) and R (25) }\end{array}$ & $\begin{array}{l}\text { NOS3 genotypes or alleles did not differ between } \\
\text { R and NR subgroups of PE or GH patients. NOS3 } \\
\text { haplotypes combining the 'C, Glu, a' alleles and 'T, Asp, } \\
\text { a' alleles were more frequent in R and NR subgroups of } \\
\text { PE patients, respectively }\end{array}$ & [14] \\
\hline MMP9 & $\begin{array}{l}\mathrm{C}-1562 \mathrm{~T}(\mathrm{rs} 3918242) \\
-90(\mathrm{CA})_{13-25}(\mathrm{rs} 2234681)\end{array}$ & $\begin{array}{l}\text { PE: R (114) and NR (99); } \\
\text { GH: R (159) and NR (22) }\end{array}$ & $\begin{array}{l}\text { MMP9 haplotypes combining the ' } \mathrm{C}, \mathrm{H} \text { ' and the ' } \mathrm{T}, \mathrm{H} \text { ' } \\
\text { alleles were more frequent in the } \mathrm{NR} \text { subgroup of } \mathrm{PE} \\
\text { and GH patients, respectively }\end{array}$ & [11] \\
\hline MMP2 & $\begin{array}{l}\text { C-1306T (rs243865), } \\
\text { C-735T (rs2285053) }\end{array}$ & $\begin{array}{l}\text { PE: R (115) and NR (100); } \\
\text { GH: R (159) and NR (22) }\end{array}$ & $\begin{array}{l}\text { MMP2 genotypes or haplotypes did not differ between } \\
\mathrm{R} \text { and NR subgroups of PE or GH patients }\end{array}$ & [12] \\
\hline $\begin{array}{l}\text { TIMP1, } \\
\text { TIMP3 }\end{array}$ & $\begin{array}{l}\text { rs2070584 T>G, } \\
\text { rs9619311 T>C }\end{array}$ & $\begin{array}{l}\text { PE: R (112) and NR (93); } \\
\text { GH: R (155) and NR (23) }\end{array}$ & $\begin{array}{l}\text { The GG genotype and the G allele of the TIMP1 } \\
\text { polymorphism were associated with PE and were more } \\
\text { frequent in the NR subgroup of PE patients } \\
\text { TIMP3 polymorphism had no effects on response to } \\
\text { the antihypertensive therapy both groups }\end{array}$ & [10] \\
\hline VEGF & $\begin{array}{l}\text { C-2578A (rs699947), } \\
\text { G-634C (rs2010963) }\end{array}$ & PE: R (46) and NR (67) & $\begin{array}{l}\text { VEGF genotypes or haplotypes did not differ between } \\
\text { R and NR subgroups of PE patients }\end{array}$ & [13] \\
\hline NAMPT & $\begin{array}{l}\text { rs1319501 T>C, } \\
\text { rs3801266 A>G }\end{array}$ & $\begin{array}{l}\text { PE: R (110) and NR (95); } \\
\text { GH: R (153) and NR (21) }\end{array}$ & $\begin{array}{l}\text { The 'C, A' haplotype was more frequent in the NR } \\
\text { subgroup of PE patients and was associated with lower } \\
\text { visfatin/NAMPT levels. Interactions among NAMPT, } \\
\text { TIMP1 and MMP2 genotypes were associated with PE } \\
\text { and with NR subgroup of PE patients }\end{array}$ & [9] \\
\hline
\end{tabular}


two SNP associations which were not replicated in other cohorts, and it is more likely that these SNPs are linked with as yet unidentified causal variants [49].

The major obstacles to the identification of genetic variants that affect PE risk are the likely small influence of individual genetic loci, the complex interplay between environmental exposures and polygenic susceptibility, and the lack of replication findings from genetic association studies [46]. Most of the studies using a candidate gene approach have focused on maternal genotypes of a single or a few genetic polymorphisms [46,50,51]. However, PE is a complex genetic disorder, and it is probable that no single gene or variant will be identified as responsible for all cases of PE [15]. This scenario highlights the need to focus on more than one candidate gene, and in the interaction among polymorphisms of different genes rather than on the effect of single loci. Indeed, gene-gene interactions were postulated as an important component of complex diseases, including PE [51].

Interestingly, we have characterized interactions between VEGF and MMP9 and between MMP9 and TIMP1 genes which were associated with the susceptibility to PE [10,52]. Further analysis of gene-gene interaction may be focused on pathways relevant to the pathophysiology of PE. For example, although NOS2 gene variants may affect the susceptibility to PE [53], the nonlinear interactions between variants in the NOS2 and NOS3 genes may also have an effect on disease susceptibility, as previously shown for migraine [54]. Notably, the interplay among genes related to NO production, oxidative stress and endothelial dysfunction in PE give support for hypotheses about the underlying molecular mechanisms, which may guide further interaction analysis among functional variants affecting the expression of these genes.

The combination of genetic, epigenetic and environmental factors may contribute to the pathophysiology of PE [55]. Thus, further gene-gene interaction and epigenetic studies may elucidate the genetic susceptibility to $\mathrm{PE}$, which will help in the identification of pharmacogenomic biomarkers related to pathophysiological processes of PE [56].

\section{Pharmacogenetics of pre-eclampsia}

There is evidence for genetic polymorphisms as determinants of the susceptibility to benign and malignant gynecologic and obstetric conditions [57]. Moreover, the therapeutic effects of drugs used in reproductive and perinatal medicine are likely to be modulated by genetic factors [58]. Notably, the lack of evidence in reproductive medicine in relation to genetic variability is compounded by the fact that many drugs currently used were not specifically studied in pregnancy, and are thus used off-label [58]. Pharmacogenetics in pregnancy is still a developing field [59]. Further efforts are needed to determine whether pharmacogenetics will aid in individualizing drug therapy in pregnancy, although emerging findings from obstetric therapeutics give glimpses into future possibilities, including antihypertensive therapy [59].

Indeed, there are few studies focusing on the pharmacogenetics of the response to antihypertensive therapy in PE (Table 1). These studies examined the association of genetic polymorphisms with subgroups of patients who were responsive or nonresponsive to antihypertensive therapy [9-14]. Methyldopa was the initial antihypertensive drug of choice, followed by nifedipine, which was added in case of lack of significant response to methyldopa. Hydralazine was used only in cases of hypertensive crisis. Therefore, we examined patients according to response to methyldopa or to total antihypertensive therapy, which included methyldopa and nifedipine and/or hydralazine. The criteria of responsiveness to the antihypertensive drugs are defined in Box 1. Patients were carefully monitored for signs and symptoms of PE, with fetal surveillance and laboratory tests once a week. Women with pre-existing hypertension or other comorbidities were not included. These pharmacogenetic studies included polymorphisms that modify the expression of candidate genes related to different pathophysiological processes in PE.

\section{Gene-drugs interactions impacting the endothelial function pathway}

Among several mediators released by the endothelium, NO plays an important role in regulating endothelial function, vasodilatation and vascular remodeling [15]. $\mathrm{NO}$ is produced by the NOS3 enzyme in the cardiovascular system. Reduced expression of NOS3 gene leads to diminished NO formation, which plays a major role in the endothelial dysfunction associated with PE [25]. Moreover, PE is associated with decreased NO bioavailability, which is inversely related to serum levels of the antiangiogenic factors sFlt-1 and sEng in PE [25]. Notably, several NOS3 polymorphisms, or the combinations of their alleles into haplotypes, were associated with PE [60,61] and related to response to cardiovascular drugs [62].

The haplotypes formed by the combination of the NOS3 polymorphisms T-786C (rs2070744) in the promoter region, Glu298Asp (rs1799983) in exon 7, and a 27 bp variable number of tandem repeats (VNTRs) $\mathrm{a} / \mathrm{b}$ in intron 4 , were associated with susceptibility to $\mathrm{PE}$ [61] and with modulation of $\mathrm{NO}$ bioavailability in PE [63]. The genotypes and alleles frequencies for these NOS3 polymorphisms did not differ between the responsive and nonresponsive subgroups of patients 
with PE or GH [14]. Moreover, the NOS3 haplotype frequencies did not differ between responsive and nonresponsive patients with GH. However, NOS3 haplotypes affected the response to total antihypertensive therapy in PE. Specifically, the haplotypes combining the ' $\mathrm{C}$, Glu, a' and ' $\mathrm{T}$, Asp, a' alleles were more frequent in the responsive and in the nonresponsive subgroup of PE patients, respectively [14].

There is no evidence that methyldopa produces antihypertensive effects by mechanisms involving increased NO production. However, it was shown that several calcium channel blockers (including nifedipine) may improve endothelial function and restore NO bioavailability [64,65]. Moreover, hydralazine, which is widely used in the treatment of PE, enhanced cyclic guanosine monophosphate (cGMP, the second messenger of $\mathrm{NO}$ ) concentrations in pregnant women with $\mathrm{PE}$, thus suggesting that this drug produces its effects by activating the NO-cGMP system [66]. It is possible that nifedipine and hydralazine used to treat PE produce their effects by enhancing NO formation, thus counteracting the impaired NO bioavailability reported in PE [25]. Therefore, NOS3 polymorphisms that modulate NO synthesis could also interfere with the response to these drugs.

Visfatin, an adipokine also known as NAMPT, is considered a potential biomarker for vascular endothelial dysfunction [67]. NAMPT polymorphisms rs1319501 T>C and rs3801266 $\mathrm{A}>\mathrm{G}$ and $N A M P T$ haplotypes were shown to affect plasma visfatin/NAMPT levels in GH and the susceptibility to PE [68]. Notably, in patients with $\mathrm{PE}$ who were nonresponsive to total antihypertensive therapy, the TC+CC genotypes of the rs1319501 polymorphism and the AG+GG genotypes of the rs 3801266 polymorphism were associated with lower and higher plasma visfatin/NAMPT levels, respectively. Moreover, the haplotype combining the ' $\mathrm{C}, \mathrm{A}$ ' alleles had higher frequency and was associated with lower visfatin/NAMPT levels in this subgroup of nonresponsive patients with PE [9]. Visfatin was shown to stimulate NOS3 expression and function in endothelial cells [69]. Conversely, visfatin was shown to impair endothelium-dependent relaxation through a mechanism involving NADPH oxidase stimulation, and therefore arises as a potential player in the development of endothelial dysfunction [70]. However, further research regarding the interplay among visfatin/NAMPT, endothelial dysfunction and the response to antihypertensive therapy in PE is needed.

VEGF is an angiogenic factor with an essential role in placental vasculogenesis and angiogenesis. PE is associated with higher sFlt-1 expression, which prevents the interaction of VEGF with its receptors downregulating its biological effects [24]. Notably, haplotypes formed by the combination of the C-2578A (rs699947) and
G-634C (rs2010963) polymorphisms in the VEGF promoter region were associated with the susceptibility to PE but not to $\mathrm{GH}$ [71]. Currently, no antihypertensive drug used during pregnancy involves a mechanism of action targeting the VEGF biology. Notably, no differences were found for the distribution of $V E G F$ genotypes or haplotypes between the subgroups of patients with PE who were responsive or nonresponsive to total antihypertensive therapy. These findings suggest that the response to antihypertensive therapy in PE is not modulated by VEGF polymorphisms [13].

Gene-drugs interactions, metalloproteinases \& their inhibitors

MMPs are a family of endopeptidases that degrade the components of the extracellular matrix, which may interact with vasoactive peptides and contribute to the endothelial dysfunction in PE. Altered placental expression of MMPs may cause shallow cytotrophoblastic invasion and incomplete remodeling of the spiral arteries. Specifically, MMP-9 and MMP-2 are involved in remodeling of placental and uterine arteries [72].

MMP-9 activity is regulated at different levels, including at transcriptional level by promoter polymorphisms which affect $M M P-9$ gene expression such as the C-1562T (rs3918242) and the microsatellite $-90(\mathrm{CA})_{13-25}(\mathrm{rs} 2234681)$, as reviewed elsewhere [73]. MMP9 polymorphisms were associated with $\mathrm{GH}$, but not with PE [74]. The CT or TT genotypes for the rs3918242 polymorphism and the MMP9 haplotype combining the ' $T$, $H$ ' alleles were more frequent in $\mathrm{GH}$ patients who were nonresponsive to methyldopa or to total antihypertensive therapy. However, The $M M P 9$ haplotype combining the ' $\mathrm{C}, \mathrm{H}$ ' alleles was more frequent in $\mathrm{PE}$ patients who were nonresponsive to methyldopa or to the total antihypertensive therapy, but not in GH patients [11]. These findings suggest that $M M P 9$ haplotypes affect the response to antihypertensive therapy in $\mathrm{GH}$ and in PE.

MMP-9 activity is also regulated by its interaction with tissue inhibitors of MMPs, including TIMP-1 and TIMP-3. Of note, TIMP-1 binds to MMP-9 with high affinity [75]. We found increased plasma MMP-9 and TIMP-1 levels in GH patients and TIMP-1 levels in PE patients, but no differences in MMP-9/TIMP-1 ratios in both groups [11]. We examined the effect of the TIMP1 rs2070584 $\mathrm{T}>\mathrm{G}$ polymorphism on TIMP-1 levels, and found that PE patients with the TG genotype had higher TIMP-1 levels [10]. We also examined the effects of TIMP1 and TIMP3 polymorphisms on response to antihypertensive therapy in PE. The TIMP3 rs9619311 polymorphism had no effects on response to antihypertensive therapy in PE. Notably, the GG genotype and G allele of the 
TIMP1 rs2070584 polymorphism were associated with PE and were more common in patients with PE who were nonresponsive to total antihypertensive therapy, which included methyldopa and nifedipine and/or hydralazine [10]. These findings suggest that the TIMP1 rs2070584 polymorphism not only is associated with PE but also decreases the responses to total antihypertensive therapy in $\mathrm{PE}[10]$.

Antihypertensive drugs including calcium channel blockers (like nifedipine) were shown to affect circulating MMP levels in essential hypertensive patients [76,77]. Plasma concentrations of MMP-2 and MMP-9 were shown to be decreased in patients with essential hypertension compared with normotensive controls. MMP-9 concentrations, but not MMP-2, were increased in hypertensive patients treated with amlodipine for 6 months. In addition, MMP-2 level was increased by treatment with felodipine, whereas diltiazem had no effects on circulating MMPs [76,77]. In this context, we examined whether circulating MMP-2 and MMP-9 levels were different between patients with $\mathrm{PE}$ who were nonresponsive or responsive to antihypertensive therapy. Lower plasma MMP-9 concentrations and MMP-9/TIMP-1 ratio (an index of net MMP-9 activity) were shown in nonresponsive compared with responsive patients, thus suggesting that the most severe cases of the disease may have undergone abnormal remodeling of placental and uterine tissues [11]. Conversely, higher plasma MMP-2 concentrations and MMP-2/TIMP-2 ratio (an index of net MMP-2 activity) were shown in nonresponsive compared with responsive patients [12], thus suggesting that antihypertensive drugs may ameliorate pre-eclamptic symptoms by decreasing net MMP-2 activity.

MMP-2 activity is also regulated at transcriptional level, and the polymorphisms C-1306T (rs243865) and C-735T (rs2285053) affect MMP2 expression, as reviewed elsewhere [78]. The genotypes and haplotypes frequencies for these $M M P 2$ polymorphisms did not differ between the responsive and nonresponsive subgroups of patients with PE or GH, which suggest that $M M P 2$ polymorphisms do not affect the response to antihypertensive therapy [12].

\section{Gene-gene interactions \& antihypertensive response}

Gene-gene interactions must also be taken into account in pharmacogenomics research. Interestingly, interactions among NAMPT, TIMP1 and $M M P 2$ genotypes were found to be associated with $\mathrm{PE}$ and with responsiveness to total antihypertensive therapy in PE [9]. The GG genotype of the TIMP1 rs2070584 polymorphism was combined with the
AA or the AG genotype of the NAMPT rs3801266 polymorphism with higher frequency in the responsive or in the nonresponsive subgroups of PE patients, respectively. These combinations were significant only when combined with the CC genotype of the MMP2 rs2285053 polymorphism. These findings are obscured when single $M M P 2$ and NAMPT genotypes alone are considered, and highlight the importance of gene-gene interactions in the genetics [52] and pharmacogenetics of PE [9,10]. These genotype combinations within the NAMPT pathway were not associated with a single drug, but with the total antihypertensive therapy, including methyldopa and nifedipine and/or hydralazine. Noteworthy, gene-gene interactions within the ACE inhibitor pathway, including the PRKCA, NOS3 and BDKRB2 polymorphisms, were shown to affect the antihypertensive response to enalapril in hypertensive patients [79,80]. Further research into the molecular mechanisms underlying the interactions among NAMPT, TIMP1 and MMP2, and their relation with response to antihypertensive therapy in PE is needed.

\section{Novel approaches to pharmacogenomics research in $\mathrm{PE}$}

In vitro model as an approach to search for novel targets

Although a large subgroup of patients with $\mathrm{PE}$ is nonresponsive to antihypertensive therapy [9-14], the underlying mechanisms are unknown. These findings suggest the need for the development of new therapies, which may be guided by pharmacogenomics approaches [56]. The endothelial dysfunction in PE supports the rationale for in vitro studies which have examined the effects of incubation of plasma from patients with $\mathrm{PE}$ and healthy pregnant women on endothelial cells $[81,82]$.

Taking a step further, we studied the effects of plasma from nonresponsive and responsive patients with $\mathrm{PE}$ on endothelial gene expression in human umbilical vein endothelial cells (HUVECs) using PCR array [83]. We found differential gene expression in HUVECs incubated with plasma from nonresponsive relative to responsive patients, and used Ingenuity Pathway Analysis to identify gene networks and interactions among genes and antihypertensive drugs used in PE. Interestingly, the genes found to be downregulated or upregulated in HUVECs incubated with plasma from nonresponsive patients in the PCR array were reported as upregulated or downregulated by nifedipine and hydralazine, respectively. For example, while MMP2 was found to be upregulated, hydralazine was shown to decrease MMP2 expression [84]. Conversely, NOS3 was found to be downregulated, and nifedipine was shown 
to increase PI3K/Akt pathway, NOS3 and soluble guanylyl cyclase activities [85]. These findings suggest that plasma from nonresponsive and responsive patients with PE evoke different responses in HUVECs, and support the incubation of plasma in HUVECs as a model to study the effects of antihypertensive drugs on gene expression.

We further explored the pathway analysis to search for interactions among the genes and drugs of potential use for treatment of nonresponsive patients. We found interactions among FGFR1 and VEGFR2 and sorafenib, regorafenib and pazopanib, which are inhibitors of FGFR1 and VEGFR2 used for cancer therapy, as these drugs inhibit the angiogenesis. While PE is characterized by an antiangiogenic state, the use of these drugs is thus not supported for antihypertensive therapy in PE [83]. However, it is important to note that pathway analysis is a powerful tool to explore potential novel targets in pharmacogenomics. For example, a recent study using pathway analysis found networks enriched for differentially expressed genes upon metformin treatment, the first-line therapy for Type 2 diabetes [86]. Several genes in the networks were not previously related to gluconeogenesis or metformin, further implicating novel molecular pathways to metformin hepatic response [86].

In summary, the incubation of plasma from nonresponsive patients in HUVECs might help in the identification of novel targets for the antihypertensive therapy in PE [83]. However, these preliminary findings should be replicated. Further pathway analysis of gene expression data might advance the pharmacogenomics research in $\mathrm{PE}$.

\section{Potential novel drugs for the treatment of pre-eclampsia}

Despite the burden of PE, the only definitive treatment is delivery of the baby and placenta. The lack of effective pharmacological therapies may be attributable to a partial understanding of its pathophysiological mechanisms, an inability to predict women who will develop PE, and a paucity of robust animal models with which to test new treatments [87]. Further studies are needed to consolidate the genetic contribution for the lack of response to current or future drugs used in PE, and then pharmacogenomics will likely be considered in treatment of PE. Current efforts in identifying potential new therapeutic targets have been reviewed elsewhere [87-90]. Next, we review examples of drugs whose responsiveness may be affected by genetic polymorphisms.

The potential use of PDE-5 inhibitors such as sildenafil and vardenafil in PE has been studied. These drugs prolong the vasodilatory effects of NO on the systemic vasculature by inhibiting the breakdown of cGMP. Another possible advantage of PDE-5 inhibitors is their vasodilatory actions in uterine-placental vessels. In this context, sildenafil and vardenafil were shown to improve endothelial dysfunction in myometrial/umbilical arteries of pregnant with PE [91,92]. A randomized study with PE patients found that sildenafil administered in a slow-escalation dosing regimen did not prolong pregnancy, although there was a trend for amelioration of blood pressure and fetal growth [93]. These studies reported no maternal or fetal adverse effects, and it seems that PDE-5 inhibitors are safe during pregnancy. However, further studies are

Current antihypertensive treatment and perspectives

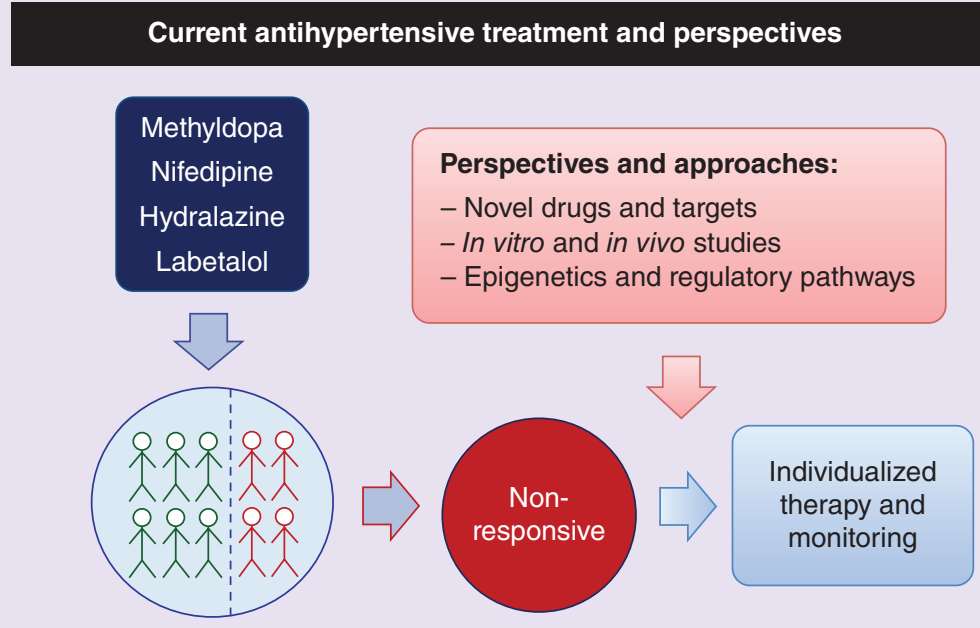

Criteria of responsiveness

Figure 1. A schematic workflow of current antihypertensive treatment and perpectives to guide further studies in the pharmacogenetic in pre-eclampsia. 
warranted to confirm their indication and posology for PE. Interestingly, genetic polymorphisms related to the NO and cGMP pathway were suggested to affect the responsiveness to therapy with PDE-5 inhibitors in erectile dysfunction [94]. This pharmacogenetic information would also need to be addressed for the treatment of PE.

Statins are also considered of potential use for the treatment of PE. A clinical study showed that pravastatin ( $40 \mathrm{mg}$, daily) stabilized blood pressure, proteinuria and circulating levels of sFlt-1, sEng and ET-1 in a cohort of preterm women with PE [95]. A recent trial provided safety and pharmacokinetics data for administration of pravastatin $(10 \mathrm{mg}$, daily) to women at high risk for PE from 12 to 17 weeks of gestation until delivery. Notably, no identifiable safety risks were noted and signs of possible efficacy were described, including restored proangiogenic profile and lower rates of $\mathrm{PE}$ and preterm delivery [96]. Although statins are contraindicated due to the risk of fetal malformations, especially in first trimester, hydrophilic statins were not associated with such risk, and the administration of pravastatin and rosuvastatin would be preferred during pregnancy. Moreover, the quality of studies reporting congenital abnormalities attributable to statins has been argued [97]. Interestingly, genetic polymorphisms may also affect the responsiveness to treatment with statins [98]. Hence, further studies are required to confirm the indication and dosage of statins for PE, and to evaluate whether treatment can be optimized based on the pharmacogenetic information.

\section{Conclusion \& future perspective}

A few studies focused on pharmacogenetics in the treatment of PE, which examined the distribution of genetic polymorphisms with subgroups of patients with $\mathrm{PE}$ who were responsive or nonresponsive to antihypertensive therapy. The chosen polymorphisms have been shown to modify the expression of candidate genes related to the pathophysiology of PE. Genotypes, haplotypes and interactions of polymorphisms were differently distributed between the responsive and nonresponsive subgroups of patients with $\mathrm{PE}$ (Table 1). Importantly, these association findings must be replicated in different populations. Moreover, further pharmacogenetic studies should be focused on other candidate genes and gene-gene interactions within pathways of relevance to the pathophysiology of PE.

It is important to note the challenges on the definition of the criteria used to assess the responsiveness to antihypertensive therapy in PE. The criteria we use in the definition of responsive and nonresponsive groups to antihypertensive therapy (Box 1) should be discussed by other researchers, including its limitations and application to different clinical scenarios and countries. For example, labetalol is not used for the treatment of HDP in Brazil. Currently, it is not clear how to precisely define the severity of HDP, and it is possible that our criteria of responsiveness denote disease severity instead. Further studies are required to improve our understanding of the HDP. However, some of the parameters included in our criteria are in agreement with the clinical criteria observed in a study regarding the effects of less-tight versus tight control of hypertension on pregnancy [99]. In this study, the pregnant women in the less-tight control group were associated with a significantly higher frequency of severe maternal hypertension and target-organ lesions $[99,100]$.

Nonresponsive patients with PE are associated with the worst clinical parameters [9-14], and could benefit from a more individualized treatment and monitoring. However, the antihypertensive drugs currently used were not specifically developed for PE. Pharmacogenomics approaches may benefit nonresponsive patients either by guiding individualized treatment and/or the identification of novel drugs for treatment of PE. Unfortunately, few research efforts have been focused on this matter to date, and the collaboration among research groups is required. Furthermore, the translation of these findings into clinical applications would be completely justifiable considering the potential reduction of both the maternal and fetal morbidity and mortality.

In addition, although a large subgroup of patients with $\mathrm{PE}$ is nonresponsive to antihypertensive therapy [9-14], the underlying mechanisms are unknown. Indeed, a small percentage of the genetic variability associated with response to antihypertensive drugs has been explained. Further studies on epigenetics and regulatory pathways related to the response to antihypertensive drugs might help to identify novel targets for antihypertensive therapy [101], including in PE. These findings suggest the need for the development of new therapies, which may be guided by pharmacogenomic approaches. For example, the incubation of plasma from pregnant women with PE in HUVECs may be an efficient model to study the effects of antihypertensive drugs on gene expression [83], and additional pathway analysis might advance the pharmacogenomics research in PE.

The perspectives that may guide the individualized therapy and the identification of novel drugs and targets for treatment of PE are shown in Figure 1. Potential therapies are currently being studied which might represent a significant positive impact on maternal and neonatal health. However, it is vital that basic research continues to identify potential mechanisms and tar- 
gets, and that any potential therapy is thoroughly tested before progression to clinical trial [87]. We expect an increase in the number of pharmacogenetic studies in the treatment of PE within the next years. The increasing knowledge of the pathophysiology of PE might shed light on novel candidate genes and path- ways, which may help in the pharmacogenetics in the treatment of PE.

Financial \& competing interests disclosure

This work was supported by grants from the National Council for Scientific and Technological Development (CNPq-

\section{Executive summary}

- Pre-eclampsia (PE) is a major cause of maternal and perinatal morbidity and mortality, defined as pregnancy-induced hypertension and proteinuria.

- Antihypertensive therapy allows the prolongation of gestation, thereby decreasing fetal and maternal adverse outcomes. However, a large subgroup of pregnant women with PE is nonresponsive to antihypertensive therapy. Pathophysiology of PE

- Although the exact mechanisms remain unclear, placental ischemia/hypoxia is implicated as a pivotal causative factor in the pathophysiology of PE.

- MMPs, nitric oxide bioavailability, oxidative stress and inflammation are mechanisms related to hypertension during pregnancy.

\section{Genetics of PE}

- Candidate gene studies are limited by the incomplete understanding of the pathophysiology of PE.

- Further gene-gene interaction and epigenetic studies may help to identify potential pharmacogenomic biomarkers.

\section{Pharmacogenetics of PE}

- A few studies examined the effects of genetic polymorphisms on response to total antihypertensive therapy in $P E$, including methyldopa and nifedipine and/or hydralazine, and considered responsive or nonresponsive patients.

Gene-drugs interactions impacting the endothelial function pathway

- NOS3 haplotypes with the 'C, Glu, a' and 'T, Asp, a' alleles of the T-786C (rs2070744), Glu298Asp (rs1799983), and 27 bp VNTR a/b polymorphisms were associated with response or lack of response in PE patients, respectively.

- NAMPT genotypes of the rs1319501 T>C and rs3801266 A>G polymorphisms affect visfatin/NAMPT levels in nonresponsive PE patients. The ' $C$, A' haplotype was associated with lower visfatin/NAMPT levels and with lack of response in PE patients.

- VEGF polymorphisms did not affect response to antihypertensive therapy in PE patients.

Gene-drugs interactions, matrix metalloproteinases \& their inhibitors

- MMP9 haplotypes with the 'C, $\mathrm{H}^{\prime}$ and ' $\mathrm{T}, \mathrm{H}$ ' alleles of the C-1562T (rs3918242) and -90(CA) ${ }_{13-25}$ (rs2234681) polymorphisms were associated with lack of response in PE and gestational hypertension patients, respectively.

- MMP2 polymorphisms did not affect response to antihypertensive therapy in PE or gestational hypertension patients.

- The GG genotype and G allele of the TIMP1 rs2070584 T>G polymorphism were associated with PE and with lack of response in PE patients.

Gene-gene interactions \& antihypertensive response

- Interactions among NAMPT, TIMP1 and MMP2 were associated with PE and with lack of response to total antihypertensive therapy in PE patients.

Novel approaches to pharmacogenomics research in PE

In vitro model as an approach to search for novel targets

- The incubation of plasma from nonresponsive and responsive patients with PE evokes different responses in human umbilical vein endothelial cells.

- This model may enable the study of the effects of antihypertensive drugs on gene expression and the identification of novel targets for PE therapy.

Potential novel drugs for the treatment of PE

- The potential use of phosphodiesterase-5 inhibitors, such as sildenafil and vardenafil, and statins are considered for the treatment of PE. However, further studies are warranted to confirm their indication and dosage for PE.

Conclusion \& future perspective

- These association findings must be replicated in different populations. Further pharmacogenetic studies should focus on other candidate genes and gene-gene interactions within pathways relevant to PE pathophysiology.

- The criteria of responsiveness to antihypertensive therapy in PE should be replicated by other researchers, including its limitations and application to different clinical scenarios and countries.

- Pharmacogenomics approaches may help the identification of novel targets and drugs, which may guide the individualized therapy of PE. 
Brazil) and the Fundação de Amparo a Pesquisa do Estado de São Paulo (FAPESP-Brazil). MR Luizon is supported by the Young Talent Attraction Fellowship from CNPq-Brazil. The authors have no other relevant affiliations or financial involvement with any organization or entity with a financial interest in or financial conflict with the subject matter or materials discussed in the manuscript apart from those disclosed.

\section{References}

Papers of special note have been highlighted as:

- of interest $\boldsymbol{\bullet} \bullet$ of considerable interest

1 Report of the National High Blood Pressure Education Program Working Group on high blood pressure in pregnancy. Am. J. Obstet. Gynecol. 183(1), S1-S22 (2000).

2 Duley L. The global impact of pre-eclampsia and eclampsia. Semin. Perinatol. 33(3), 130-137 (2009).

3 Carty DM, Delles C, Dominiczak AF. pre-eclampsia and future maternal health. J. Hypertens. 28(7), 1349-1355 (2010).

4 Chen CW, Jaffe IZ, Karumanchi SA. Pre-eclampsia and cardiovascular disease. Cardiovasc. Res. 101(4), 579-586 (2014)

5 Scantlebury DC, Hayes SN. How does pre-eclampsia predispose to future cardiovascular disease? Curr. Hypertens. Rep. 16(9), 472 (2014).

6 Umesawa M, Kobashi G. Epidemiology of hypertensive disorders in pregnancy: prevalence, risk factors, predictors and prognosis. Hypertens. Res. 40 (3), 213-220 (2017).

7 Berzan E, Doyle R, Brown CM. Treatment of pre-eclampsia: current approach and future perspectives. Curr. Hypertens. Rep. 16(9), 473 (2014).

8 Podymow T, August P. Update on the use of antihypertensive drugs in pregnancy. Hypertension 51(4), 960-969 (2008).

9 Luizon MR, Palei AC, Belo VA et al. Gene-gene interactions in the NAMPT pathway, plasma visfatin/NAMPT levels, and antihypertensive therapy responsiveness in hypertensive disorders of pregnancy. Pharmacogenomics J. doi:10.1038/ tpj.2016.35 (2016) (Epub ahead of print).

-• $\quad$ First study that found interactions among NAMPT, TIMPI and $M M P 2$ genotypes associated with susceptibility to pre-eclampsia $(\mathrm{PE})$, and with lack of response to antihypertensive therapy in PE. These findings highlight the importance of considering gene-gene interactions in the pharmacogenetics of PE.

10 Luizon MR, Palei AC, Sandrim VC et al. Tissue inhibitor of matrix metalloproteinase-1 polymorphism, plasma TIMP-1 levels, and antihypertensive therapy responsiveness in hypertensive disorders of pregnancy. Pharmacogenomics $J$. 14(6), 535-541 (2014).

- The TIMP1 rs2070584 polymorphism was associated with susceptibility to PE and with lack of response to total antihypertensive therapy in PE. Moreover, interactions between TIMP1 and MMP9 were associated with PE.
No writing assistance was utilized in the production of this manuscript.

\section{Open access}

This work is licensed under the Attribution-NonCommercial-NoDerivatives 4.0 Unported License. To view a copy of this license, visit http://creativecommons.org/licenses/bync-nd/4.0/

11 Palei AC, Sandrim VC, Amaral LM et al. Matrix metalloproteinase-9 polymorphisms affect plasma MMP-9 levels and antihypertensive therapy responsiveness in hypertensive disorders of pregnancy. Pharmacogenomics J. 12(6), 489-498 (2012).

- $\quad$ Found that MMP9 haplotypes were associated with lack of response to methyldopa or to total antihypertensive therapy in $\mathrm{PE}$ and in gestational hypertension.

12 Palei AC, Sandrim VC, Amaral LM et al. Effects of matrix metalloproteinase $(M M P)-2$ polymorphisms on responsiveness to antihypertensive therapy of women with hypertensive disorders of pregnancy. Basic Clin. Pharmacol. Toxicol. 111(4), 262-267 (2012)

13 Sandrim VC, Palei AC, Eleuterio N, Tanus-Santos JE, Cavalli RC. Antihypertensive therapy in pre-eclampsia is not modulated by VEGF polymorphisms. Arch. Gynecol. Obstet. 291(4), 799-803 (2015).

14 Sandrim VC, Palei AC, Luizon MR, Izidoro-Toledo TC, Cavalli RC, Tanus-Santos JE. eNOS haplotypes affect the responsiveness to antihypertensive therapy in pre-eclampsia but not in gestational hypertension. Pharmacogenomics J. 10(1), 40-45 (2010).

-. First study that segregated PE patients into responsive and nonresponsive to antihypertensive therapy. NOS3 haplotypes affected the response to total antihypertensive therapy in PE and were more informative than genotypes.

15 Williams PJ, Morgan L. The role of genetics in pre-eclampsia and potential pharmacogenomic interventions. Pharmgenomics Pers. Med. 5, 37-51 (2012).

-. In this review, the authors discussed key factors in the development of PE, treatment and prevention of PE, the benefits of pharmacogenomics and present the nitric oxide synthase as a potential target for therapy of PE.

16 Gilbert JS, Ryan MJ, Lamarca BB, Sedeek M, Murphy SR, Granger JP. Pathophysiology of hypertension during pre-eclampsia: linking placental ischemia with endothelial dysfunction. Am. J. Physiol. Heart Circ. Physiol. 294(2), H541-H550 (2008).

17 Roberts JM, Hubel CA. The two stage model of preeclampsia: variations on the theme. Placenta 30 (Suppl. A), S32-S37 (2009)

18 Whitley GS, Cartwright JE. Cellular and molecular regulation of spiral artery remodelling: lessons from the cardiovascular field. Placenta 31(6), 465-474 (2010).

19 Lyall F, Robson SC, Bulmer JN. Spiral artery remodeling and trophoblast invasion in pre-eclampsia and fetal growth 
restriction: relationship to clinical outcome. Hypertension 62(6), 1046-1054 (2013).

arca BD, Gilbert J, Granger JP. Recent progress toward the understanding of the pathophysiology of hypertension during pre-eclampsia. Hypertension 51(4), 982-988 (2008).

21 Warrington JP, George EM, Palei AC, Spradley FT, Granger JP. Recent advances in the understanding of the pathophysiology of pre-eclampsia. Hypertension 62(4), 666-673 (2013).

22 Maynard SE, Karumanchi SA. Angiogenic factors and preeclampsia. Semin. Nephrol. 31(1), 33-46 (2011). circulating antiangiogenic factors in pre-eclampsia. $N$. Engl. J. Med. 355(10), 992-1005 (2006)

24 Maynard SE, Min JY, Merchan J et al. Excess placental soluble fms-like tyrosine kinase 1 (sFlt1) may contribute to endothelial dysfunction, hypertension, and proteinuria in pre-eclampsia. J. Clin. Invest. 111(5), 649-658 (2003).

25 Sandrim VC, Palei AC, Metzger IF, Gomes VA, Cavalli RC, Tanus-Santos JE. Nitric oxide formation is inversely related to serum levels of antiangiogenic factors soluble fms-like tyrosine kinase- 1 and soluble endogline in pre-eclampsia. Hypertension 52 (2), 402-407 (2008). in antiangiogenic factors in early pregnancy and risk of developing pre-eclampsia. Hypertension 50(1), 137-142 (2007).

27 Robinson CJ, Johnson DD. Soluble endoglin as a secondtrimester marker for pre-eclampsia. Am. J. Obstet. Gynecol. 197(2), 174. e1-174. e5 (2007).

28 Redman CW, Tannetta DS, Dragovic RA et al. Review: does size matter? Placental debris and the pathophysiology of preeclampsia. Placenta 33(Suppl.) S48-S54 (2012).

29 Lamarca B, Cornelius D, Wallace K. Elucidating immune mechanisms causing hypertension during pregnancy. Physiology (Bethesda) 28(4), 225-233 (2013).

30 Freeman DJ, Mcmanus F, Brown EA et al. Short- and longterm changes in plasma inflammatory markers associated with pre-eclampsia. Hypertension 44(5), 708-714 (2004).

31 Sharma A, Satyam A, Sharma JB. Leptin, IL-10 and inflammatory markers (TNF-alpha, IL-6 and IL-8) in preeclamptic, normotensive pregnant and healthy non-pregnant women. Am. J. Reprod. Immunol. 58(1), 21-30 (2007).

32 Herse F, Lamarca B. Angiotensin II type 1 receptor autoantibody (AT1-AA)-mediated pregnancy hypertension. Am. J. Reprod. Immunol. 69(4), 413-418 (2013).

33 Xia Y, Kellems RE. Angiotensin receptor agonistic autoantibodies and hypertension: pre-eclampsia and beyond. Circ. Res. 113(1), 78-87 (2013).

34 Hladunewich M, Karumanchi SA, Lafayette R. Pathophysiology of the clinical manifestations of preeclampsia. Clin. J. Am. Soc. Nephrol. 2(3), 543-549 (2007).

35 Aydin S, Benian A, Madazli R, Uludag S, Uzun H, Kaya S. Plasma malondialdehyde, superoxide dismutase, sE-selectin, fibronectin, endothelin-1 and nitric oxide levels in women with pre-eclampsia. Eur. J. Obstet. Gynecol. Reprod. Biol. 113(1), 21-25 (2004).
36 Nova A, Sibai BM, Barton JR, Mercer BM, Mitchell MD. Maternal plasma level of endothelin is increased in preeclampsia. Am. J. Obstet. Gynecol. 165(3), 724-727 (1991).

37 Taylor RN, Varma M, Teng NN, Roberts JM. Women with pre-eclampsia have higher plasma endothelin levels than women with normal pregnancies. J. Clin. Endocrinol. Metab. 71(6), 1675-1677 (1990).

38 Lankhorst S, Kappers MH, Van Esch JH, Danser AH, Van Den Meiracker AH. Hypertension during vascular endothelial growth factor inhibition: focus on nitric oxide, endothelin-1, and oxidative stress. Antioxid. Redox Signal. 20(1), 135-145 (2014).

39 Rodrigo R, Gonzalez J, Paoletto F. The role of oxidative stress in the pathophysiology of hypertension. Hypertens. Res. 34(4), 431-440 (2011).

40 Shokry M, Omran OM, Hassan HI, Elsedfy GO, Hussein MR. Expression of matrix metalloproteinases 2 and 9 in human trophoblasts of normal and preeclamptic placentas: preliminary findings. Exp. Mol. Pathol. 87(3), 219-225 (2009).

41 Fernandez-Patron C, Radomski MW, Davidge ST. Vascular matrix metalloproteinase-2 cleaves big endothelin-1 yielding a novel vasoconstrictor. Circ. Res. 85(10), 906-911 (1999).

42 Fernandez-Patron C, Stewart KG, Zhang Y, Koivunen E, Radomski MW, Davidge ST. Vascular matrix metalloproteinase-2-dependent cleavage of calcitonin generelated peptide promotes vasoconstriction. Circ. Res. 87(8), 670-676 (2000)

43 Martinez A, Oh HR, Unsworth EJ et al. Matrix metalloproteinase- 2 cleavage of adrenomedullin produces a vasoconstrictor out of a vasodilator. Biochem. J. 383(Pt. 3), 413-418 (2004).

44 Tran ED, Delano FA, Schmid-Schonbein GW. Enhanced matrix metalloproteinase activity in the spontaneously hypertensive rat: VEGFR-2 cleavage, endothelial apoptosis, and capillary rarefaction. J. Vasc. Res. 47(5), 423-431 (2010).

45 Tran ED, Yang M, Chen A, Delano FA, Murfee WL, Schmid-Schonbein GW. Matrix metalloproteinase activity causes VEGFR-2 cleavage and microvascular rarefaction in rat mesentery. Microcirculation 18(3), 228-237 (2011).

46 Staines-Urias E, Paez MC, Doyle P et al. Genetic association studies in pre-eclampsia: systematic meta-analyses and field synopsis. Int. J. Epidemiol. 41(6), 1764-1775 (2012).

47 Zhao L, Bracken MB, Dewan AT. Genome-wide association study of pre-eclampsia detects novel maternal single nucleotide polymorphisms and copy-number variants in subsets of the Hyperglycemia and Adverse Pregnancy Outcome (HAPO) study cohort. Ann. Hum. Genet. 77(4), 277-287 (2013).

48 Zhao L, Triche EW, Walsh KM et al. Genome-wide association study identifies a maternal copy-number deletion in PSG11 enriched among pre-eclampsia patients. $B M C$ Pregnancy Childbirth 12, 61 (2012).

49 Johnson MP, Brennecke SP, East CE et al. Genome-wide association scan identifies a risk locus for pre-eclampsia on 2q14, near the inhibin, beta B gene. PLoS ONE 7(3), e33666 (2012). 
50 Chappell S, Morgan L. Searching for genetic clues to the causes of pre-eclampsia. Clin. Sci. (Lond.) 110(4), 443-458 (2006).

51 Williams PJ, Broughton Pipkin F. The genetics of preeclampsia and other hypertensive disorders of pregnancy. Best Pract. Res. Clin. Obstet. Gynaecol. 25(4), 405-417 (2011).

52 Luizon MR, Sandrim VC, Palei AC et al. Epistasis among eNOS, MMP-9 and VEGF maternal genotypes in hypertensive disorders of pregnancy. Hypertens. Res. 35(9), 917-921 (2012).

53 Amaral LM, Palei AC, Sandrim VC et al. Maternal iNOS genetic polymorphisms and hypertensive disorders of pregnancy. J. Hum. Hypertens. 26(9), 547-552 (2012).

54 Goncalves FM, Luizon MR, Speciali JG, Martins-Oliveira A, Dach F, Tanus-Santos JE. Interaction among nitric oxide (NO)-related genes in migraine susceptibility. Moll. Cell. Biochem. 370 (1-2), 183-189 (2012).

55 Chelbi ST, Vaiman D. Genetic and epigenetic factors contribute to the onset of pre-eclampsia. Mol. Cell. Endocrinol. 282(1-2), 120-129 (2008).

56 Luizon MR, Sandrim VC. Pharmacogenomic approaches that may guide pre-eclampsia therapy. Pharmacogenomics 14(6), 591-593 (2013).

57 Tempfer CB, Schneeberger C, Huber JC. Applications of polymorphisms and pharmacogenomics in obstetrics and gynecology. Pharmacogenomics 5(1), 57-65 (2004).

58 Alfirevic A, Alfirevic Z, Pirmohamed M. Pharmacogenetics in reproductive and perinatal medicine. Pharmacogenomics 11(1), 65-79 (2010).

59 Haas DM. Pharmacogenetics and individualizing drug treatment during pregnancy. Pharmacogenomics 15(1), 69-78 (2014).

-. In this review, the author summarized data regarding several areas of obstetric therapeutics, and highlighted research needs to determine if pharmacogenetics will aid in individualizing therapy in pregnancy.

60 Muniz L, Luizon MR, Palei AC et al. eNOS tag SNP haplotypes in hypertensive disorders of pregnancy. DNA Cell Biol. 31(12), 1665-1670 (2012).

61 Sandrim VC, Palei AC, Cavalli RC et al. eNOS haplotypes associated with gestational hypertension or pre-eclampsia. Pharmacogenomics 9(10), 1467-1473 (2008).

62 Oliveira-Paula GH, Lacchini R, Tanus-Santos JE. Clinical and pharmacogenetic impact of endothelial nitric oxide synthase polymorphisms on cardiovascular diseases. Nitric Oxide 63, 39-51 (2017).

63 Sandrim VC, Palei AC, Sertorio JT, Cavalli RC, Duarte G, Tanus-Santos JE. Effects of eNOS polymorphisms on nitric oxide formation in healthy pregnancy and in pre-eclampsia. Mol. Hum. Reprod. 16(7), 506-510 (2010).

64 Ding Y, Vaziri ND. Nifedipine and diltiazem but not verapamil up-regulate endothelial nitric-oxide synthase expression. J. Pharmacol. Exp. Ther. 292(2), 606-609 (2000).

65 Taddei S, Virdis A, Ghiadoni L et al. Restoration of nitric oxide availability after calcium antagonist treatment in essential hypertension. Hypertension 37(3), 943-948 (2001).

66 Lopez-Jaramillo P, Narvaez M, Calle A et al. Cyclic guanosine 3', 5' monophosphate concentrations in pre-eclampsia: effects of hydralazine. Br. J. Obstet. Gynaecol. 103(1), 33-38 (1996).

67 Peiro C, Romacho T, Carraro R, Sanchez-Ferrer CF. Visfatin/PBEF/NAMPT: a new cardiovascular target? Front. Pharmacol. 1, 135 (2010).

68 Luizon MR, Belo VA, Palei AC et al. Effects of NAMPT polymorphisms and haplotypes on circulating visfatin/ NAMPT levels in hypertensive disorders of pregnancy. Hypertens. Res. 38(5), 361-366 (2015).

69 Lovren F, Pan Y, Shukla PC et al. Visfatin activates eNOS via Akt and MAP kinases and improves endothelial cell function and angiogenesis in vitro and in vivo: translational implications for atherosclerosis. Am. J. Physiol. Endocrinol. Metab. 296(6), E1440-E1449 (2009).

70 Vallejo S, Romacho T, Angulo J et al. Visfatin impairs endothelium-dependent relaxation in rat and human mesenteric microvessels through nicotinamide phosphoribosyltransferase activity. PLoS ONE 6(11), e27299 (2011).

71 Sandrim VC, Palei AC, Cavalli RC et al. Vascular endothelial growth factor genotypes and haplotypes are associated with pre-eclampsia but not with gestational hypertension. Mol. Hum. Reprod. 15(2), 115-120 (2009).

72 Isaka $\mathrm{K}$, Usuda S, Ito $\mathrm{H}$ et al. Expression and activity of matrix metalloproteinase 2 and 9 in human trophoblasts. Placenta 24(1), 53-64 (2003).

73 Metzger IF, Luizon MR, Lacchini R, Tanus-Santos JE. Genetic variants in matrix metalloproteinase-9 gene modify metalloproteinase-9 levels in black subjects. DNA Cell Biol. 31(4), 504-510 (2012).

74 Palei AC, Sandrim VC, Duarte G, Cavalli RC, Gerlach RF, Tanus-Santos JE. Matrix metalloproteinase (MMP)-9 genotypes and haplotypes in pre-eclampsia and gestational hypertension. Clin. Chim. Acta 411(11-12), 874-877 (2010).

75 Van Den Steen PE, Dubois B, Nelissen I, Rudd PM, Dwek RA, Opdenakker G. Biochemistry and molecular biology of gelatinase B or matrix metalloproteinase-9 (MMP-9). Crit. Rev. Biochem. Mol. Biol. 37(6), 375-536 (2002).

76 Zervoudaki A, Economou E, Pitsavos $\mathrm{C}$ et al. The effect of $\mathrm{Ca}^{2+}$ channel antagonists on plasma concentrations of matrix metalloproteinase- 2 and -9 in essential hypertension. Am. J. Hypertens. 17(3), 273-276 (2004).

77 Zervoudaki A, Economou E, Stefanadis C et al. Plasma levels of active extracellular matrix metalloproteinases 2 and 9 in patients with essential hypertension before and after antihypertensive treatment. J. Hum. Hypertens. 17(2), 119-124 (2003).

78 Lacchini R, Jacob-Ferreira AL, Luizon MR et al. Common matrix metalloproteinase 2 gene haplotypes may modulate left ventricular remodelling in hypertensive patients. J. Hum. Hypertens 26(3), 171-177 (2012).

79 Oliveira-Paula GH, Luizon MR, Lacchini R et al. Gene-gene interactions among PRKCA, NOS3 and BDKRB2 polymorphisms affect the antihypertensive effects of enalapril. Basic Clin. Pharmacol. Toxicol. 120(3), 284-291 (2017). 
Silva PS, Fontana V, Luizon MR et al. eNOS and BDKRB2 genotypes affect the antihypertensive responses to enalapril. Eur. J. Clin. Pharmacol. 69(2), 167-177 (2013).

81 Mackenzie RM, Sandrim VC, Carty DM et al. Endothelial FOS expression and pre-eclampsia. BJOG 119(13), 1564-1571 (2012).

82 Sankaralingam S, Lalu MM, Xu Y, Davidge ST. Effect of peroxynitrite scavenging on endothelial cells stimulated by plasma from women with pre-eclampsia: a proteomic approach. Hypertens. Pregnancy 29(4), 419-428 (2010).

83 Luizon MR, Caldeira-Dias M, Deffune E et al. Antihypertensive therapy in pre-eclampsia: effects of plasma from nonresponsive patients on endothelial gene expression. Pharmacogenomics 17(10), 1121-1127 (2016).

-• $\quad$ First study showing different effects of plasma from nonresponsive and responsive patients with $\mathrm{PE}$ on endothelial gene expression in human umbilical vein endothelial cells. This in vitro model may be used to study the effects of antihypertensive drugs on gene expression and to identifiy novel targets for therapy of PE.

84 Kodavanti UP, Thomas RF, Ledbetter AD et al. Diesel exhaust induced pulmonary and cardiovascular impairment: the role of hypertension intervention. Toxicol. Appl. Pharmacol. 268(2), 232-240 (2013).

85 Shih CY, Lin MH, Fan HC, Chen FC, Chou TC. Mechanisms of antiplatelet activity of nifedipine: role of peroxisome proliferator-activated receptor-beta-gammadependent processes. J. Hypertens. 32(1), 181-192 (2014).

86 Luizon MR, Eckalbar WL, Wang Y et al. Genomic characterization of metformin hepatic response. PLoS Genet. 12(11), e1006449 (2016).

87 Oyston CJ, Stanley JL, Baker PN. Potential targets for the treatment of pre-eclampsia. Expert. Opin. Ther. Targets 19(11), 1517-1530 (2015).

88 Downing JW, Baysinger CL, Johnson RF, Paschall RL. Review: potential druggable targets for the treatment of early onset preeclampsia. Pregnancy Hypertens. 3(4), 203-210 (2013).

89 George EM. New approaches for managing pre-eclampsia: clues from clinical and basic research. Clin. Ther. 36(12), 1873-1881 (2014).

90 Palei AC, Granger JP, Tanus-Santos JE. Matrix metalloproteinases as drug targets in pre-eclampsia. Curr. Drug Targets 14(3), 325-334 (2013).
91 Karasu E, Kayacan N, Sadan G, Dinc B. Different effects of different phosphodiesterase type-5 inhibitors in preeclampsia. Pregnancy Hypertens. 1(3-4), 231-237 (2011).

92 Wareing M, Myers JE, O’Hara M et al. Effects of a phosphodiesterase-5 (PDE5) inhibitor on endotheliumdependent relaxation of myometrial small arteries. Am. J. Obstet. Gynecol. 190(5), 1283-1290 (2004).

93 Samangaya RA, Mires G, Shennan A et al. A randomised, double-blinded, placebo-controlled study of the phosphodiesterase type 5 inhibitor sildenafil for the treatment of pre-eclampsia. Hypertens. Pregnancy 28(4), 369-382 (2009)

94 Lacchini R, Tanus-Santos JE. Pharmacogenetics of erectile dysfunction: navigating into uncharted waters. Pharmacogenomics 15(11), 1519-1538 (2014).

95 Brownfoot FC, Tong S, Hannan NJ et al. Effects of pravastatin on human placenta, endothelium, and women with severe pre-eclampsia. Hypertension 66(3), 687-697; discussion 445 (2015).

96 Costantine MM, Cleary K, Hebert MF et al. Safety and pharmacokinetics of pravastatin used for the prevention of pre-eclampsia in high-risk pregnant women: a pilot randomized controlled trial. Am. J. Obstet. Gynecol. 214(6), 720.e1-720.e17 (2016).

97 Morton S, Thangaratinam S. Statins in pregnancy. Curr. Opin. Obstet. Gynecol. 25(6), 433-440 (2013).

$98 \mathrm{Hu}$ M, Tomlinson B. Pharmacogenomics of lipid-lowering therapies. Pharmacogenomics 14(8), 981-995 (2013).

99 Magee LA, Von Dadelszen P, Rey E et al. Less-tight versus tight control of hypertension in pregnancy. N. Engl. J. Med. 372(5), 407-417 (2015).

-. Clinical study on the effects of 'less-tight' versus 'tight' control of hypertension on pregnancy. The 'lesstight' group was associated with a significantly higher frequency of severe hypertension and target-organ lesions.

100 Magee LA, Von Dadelszen P, Singer J et al. The CHIPS randomized controlled trial (control of hypertension in pregnancy study): is severe hypertension just an elevated blood pressure? Hypertension 68(5), 1153-1159 (2016).

101 Fontana V, Luizon MR, Sandrim VC. An update on the pharmacogenetics of treating hypertension. J. Hum. Hypertens. 29(5), 283-291 (2015). 